\title{
Endovascular Revascularization in Secondary Raynaud's Phenomenon
}

\author{
Pim W van Egmond ${ }^{\mathrm{a}}$, Peter M. T. Pattynama ${ }^{\mathrm{b}}$, Peter M Schlejen ${ }^{\mathrm{a}, \mathrm{c}}$
}

\begin{abstract}
Endovascular intervention is not previously described in patients with exacerbated Raynaud's phenomenon (RP). A 45-year-old male with secondary RP and CREST (Calcinosis cutis, Raynaud's phenomenon, Esophageal dysmotility, Sclerodactyly and Telangiectasia) with a smoking history, who was noncompliant in the noninterventional treatment, presented with necrosis of the right hand in exacerbated RP. An intra-arterial digital subtraction angiography (DSA) showed occlusion of the right distal ulnar and radial arteries. Initial pharmacological therapy showed no improvement. Thrombolysis with Urokinase therapy had a beneficial, but not optimal, effect on perfusion. This was probably due to an atherosclerotic component. An endovascular intervention, $2 \mathrm{~mm}$ balloon dilatation, in the right radial artery; improved this significantly. The clinical situation proved stable and no expansion of necrosis was seen. An endovascular procedure, not previously described in RP management, can be helpful in the treatment of exacerbated RP; especially when an atherosclerotic component is present.
\end{abstract}

Keywords: Endovascular; Necrosis; CREST; Secondary Raynaud's phenomenon

\section{Introduction}

Raynaud's phenomenon can manifest itself as an isolated, acute or sub acute disorder and is characterized by pallor, cyanosis and redness with pain. RP is caused by an episodic vasospasm of the peripheral arteries. Primary, or idiopathic,

Manuscript accepted for publication December 14, 2011

${ }^{a}$ Department of Surgery, Groene Hart Ziekenhuis Gouda, The Netherlands

${ }^{\mathrm{b}}$ Department of Radiology, Groene Hart Ziekenhuis Gouda, The Netherlands

${ }^{\mathrm{c}}$ Corresponding author: Peter M Schlejen, Department of Surgery, PO

Box 1098, 2803 HH, Gouda, The Netherlands.

Email: peter.schlejen@ghz.nl

doi: http://dx.doi.org/10.4021/jmc478w
RP (Raynaud's disease) occurs without an underlying disease and lacks complication of ischemic damage. Secondary RP (Raynaud's syndrome) occurs with a related illness, such as systemic lupus erythematosus or systemic sclerosis (SSc) and may lead to ulceration or gangrene. Patients with limited cutaneous systemic sclerosis (lcSSc), a subdivision of SSc, typically have prominent vascular manifestations.

\section{Case Report}

\section{Case 1}

A 45-year-old male was presented to our Accident and Emergency Department with exacerbation of Raynaud's phenomenon (RP). His right hand turned purple the evening before. No pain was felt. In 1987 this patient was diagnosed with $\mathrm{RP}$ and 10 years later with lcSSc and CREST syndrome. Although vasodilatory medication was prescribed he stopped taking these a half year ago. He smoked one pack of cigarettes each week (5 pack years). His further medical history showed a chemical sympathectomy in the left foot and a sliding hernia diaphragmatic.

With physical examination his right hand was notably purple, sclerodactyly was seen and the distal phalanges were paler. The capillary refill on the right side was prolonged. Both the ulnar and radial arteries were palpable. No necrosis or ulcera were observed.

With the diagnosis exacerbation of RP, medication (calcium channel blockers and other vasodilators; sympatholytic agents, prostaglandins) were prescribed and he was strongly advised to stop smoking. Appointments were made for the rheumatologist and the vascular surgeon.

\section{Case 2}

Two weeks later he was admitted by the rheumatologist in our hospital with necrosis on the radial side of the distal phalanx from digitus I and II, of the right hand, and ischemia of the other fingers. Blood test showed the following: CRP $8 \mathrm{mg} / \mathrm{L}$; ESR $25 \mathrm{~mm} / \mathrm{hr}$; Thrombocytes $330 \times 10^{9} / \mathrm{L}$; Leukocytes $11.1 \times 10^{9} / \mathrm{L}$; ANA positive; anti-ENA positive, 


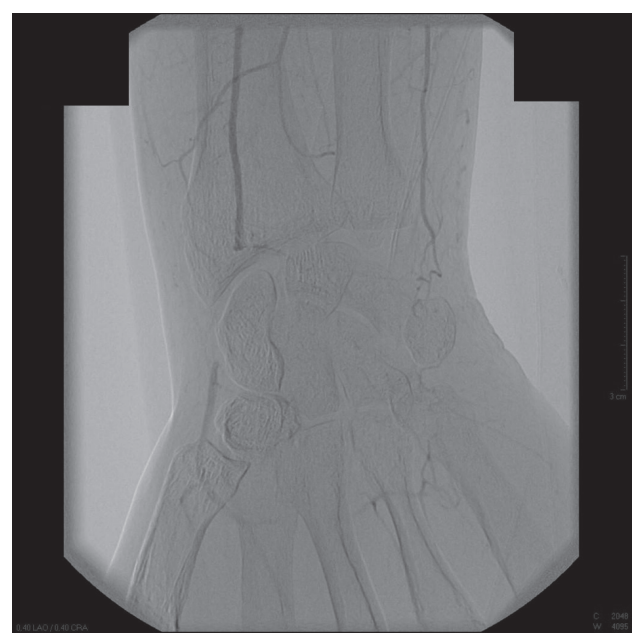

Figure 1. An intra-arterial digital subtraction angiography on the day of second presentation. Occlusion of the right distal radial and ulnar arteries is seen.

ANF positive and anti-centromere antibodies positive. On an electrocardiogram and ultrasound of the heart no cardiac embolism source could be found. Treatment with intravenous Iloprost was started but showed no improvement. An intra-arterial DSA showed occlusion of the right distal ulnar

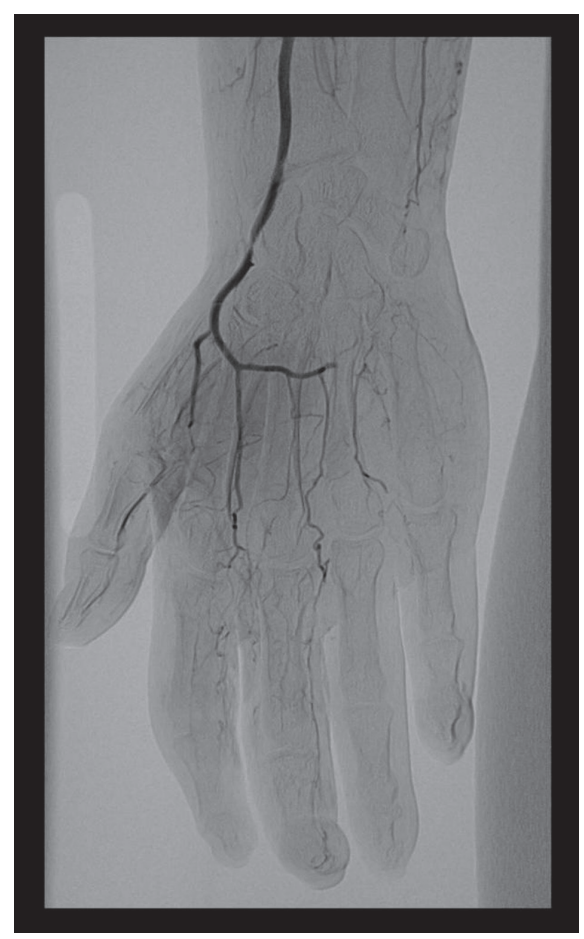

Figure 2. An intra-arterial digital subtraction angiography after three days of thrombolysis therapy. A local stenosis just above the styloideus radii is visible in the radial artery. The ulnar artery remains hypoplastic.

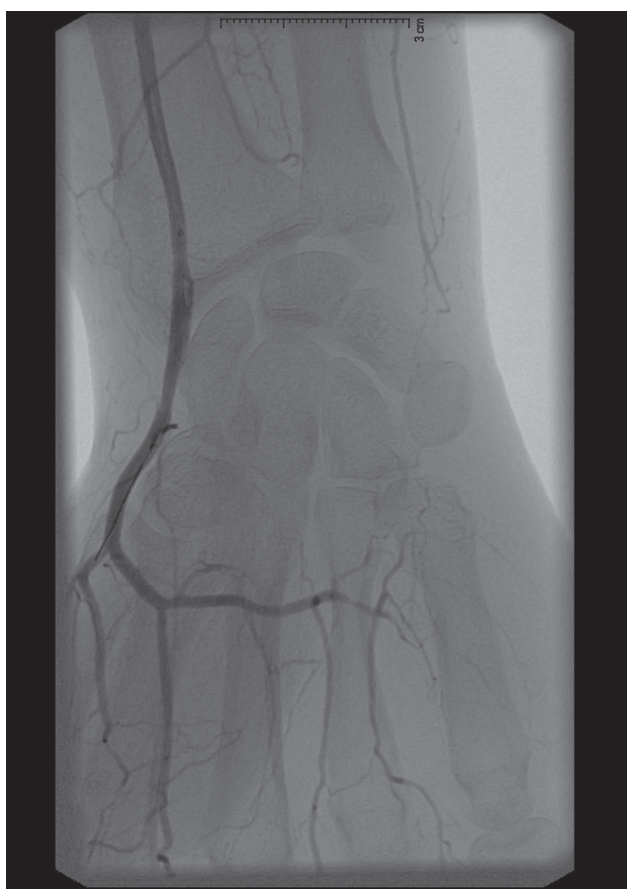

Figure 3. With a percutaneous transluminal arterial (PTA) angiography the radial artery was dilated, the catheter is visible.

and radial arteries. Through the collateral circuit, the right palmar arcade became partially visible (Fig. 1). No corkscrew appearances, as in Buerger's disease, were seen on angiographs. This absent finding, the medical history of this patient and the fact he did not have a cigarette the day of second presentation makes this disease less likely. Vascularization did not improve after vasodilatation with Tolazoline, so thrombolysis with Urokinase (bolus of $250,000 \mathrm{U}$ and $100,000 \mathrm{U} / \mathrm{hr}$ ) was initiated.

In the next two days the angiography showed improvement of the collateral circuit. The radial artery was still occluded over a distance of $3 \mathrm{~cm}$, which improved to a local stenosis just above the styloideus radii. The ulnar artery proved to be hypoplastic; thus the vascular arcade of the right hand remained incomplete. The Urokinase therapy was terminated because no further improvement was expected (Fig. 2). It was then attempted to improve perfusion of the hand and fingers by dilatation of the high-grade stenosis in the radial artery. Successful PTA was performed by using a $2 \mathrm{~mm}$ diameter balloon and by using an antegrade puncture of the brachial artery (Fig. 3). There have been no earlier reports in the literature on using PTA in patients with exacerbated RP.

On physical examination the right hand was warm and the (dry) necrosis did not increase, the pain decreased. Medication was prescribed and the patient was discharged. Appointments were made for the rheumatologist and the vas- 
cular surgeon. At follow up the necrosis of digitus II and III were treated respectively with a surgical debridement and amputation of the distal phalanx. At seven months follow up the peripheral wounds were healed, peripheral blood flow was good and the radial artery was manually palpable.

\section{Discussion}

Systemic sclerosis is a multiorgan disease with wide-spread fibrosis, activation of the immune system and extensive vascular damage [1]. SSc is generally subdivided into limited and diffuse cutaneous subsets. Patients with lcSSc typically have skin sclerosis restricted to the hands, face and neck. Also, vascular manifestations are prominent in patients with lcSSc. RP, the earliest manifestation of vascular involvement, is present in $95 \%$ of patients with SSc [2]. The pathogenesis of secondary RP is not entirely known but in short is due to endothelial cell injury in micro vessels, structural and biochemical changes of the vessel wall with progressive luminal narrowing and eventually occlusion [3-7]. Because patients with SSc have a defective angiogenesis and vasculogenesis; the loss of capillaries is not compensated $[8,9]$. Also, a high prevalence of angiographic abnormalities compatible with atherosclerotic vascular disease and hypercholesterolemia have been found in patients with Raynaud's, maybe contributing to the pathogenesis of RP [10].

Treatment of RP consists of life-style advice, drug therapy (calcium channel blockers and other vasodilators, sympatholytic agents, prostaglandins), anticoagulation and antithrombotic therapy [11]. Surgical therapy includes arterial bypass, digital arterial reconstruction, sympathectomy (peripheral and digital) and amputation [12-15]. Recently botulinum toxin injection and local or intramuscular injection of mesenchymal stem cell have been used in the management of patients with digital ischemia due to secondary RP [1618]. An endovascular procedure, as we present in our case, has not been reported previously in the management of RP.

Management of secondary RP consists mainly of drugs (calcium antagonists, vasodilators, alpha-adrenergic receptor blockers and drugs that interfere with sympathetic nerve activity). In a recent systematic review regarding the effectiveness of interventions for secondary RP clear evidence was found in favour of calcium channel blockers and (oral and intravenous) Iloprost. No studies could be found on the effectiveness of surgical interventions [13].

In this case report we showed a noncompliant patient with CREST syndrome and an exacerbation of his RP. Pharmacological therapy, Iloprost included, showed no improvement. An angiography showed extreme vasospasm with, probably, secondary thrombosis. Because of the smoking history, an atherosclerotic component could be involved.

With thrombolytic therapy the radial artery, and palmar arcade, could be revascularized. An endovascular procedure, not previously described in RP management, in the same artery helped save the right hand. This approach can be helpful in the treatment of patients with an exacerbated RP not responding on conventional therapy; especially when an atherosclerotic component is present. At 7 months follow up this proved to be an effective treatment strategy which can be safely conducted as a last resort in patients with exacerbated secondary RP.

\section{Sources of Funding}

None.

\section{Conflict of Interest}

The authors of this article do not have any financial or personal relationships with other people, or organisations, that could inappropriately influence this article. There are no conflicts of interest. The paper is not based on a previous communication to a society or meeting.

\section{References}

1. Wigley FM. Vascular disease in scleroderma. Clin Rev Allergy Immunol. 2009;36(2-3):150-175.

2. Steen V, Denton CP, Pope JE, Matucci-Cerinic M. Digital ulcers: overt vascular disease in systemic sclerosis. Rheumatology (Oxford). 2009;48(Suppl 3):iii19-24.

3. Hasegawa M, Nagai Y, Tamura A, Ishikawa O. Arteriographic evaluation of vascular changes of the extremities in patients with systemic sclerosis. Br J Dermatol. 2006;155(6):1159-1164.

4. Geyer M, Muller-Ladner U. The pathogenesis of systemic sclerosis revisited. Clin Rev Allergy Immunol. 2011;40(2):92-103.

5. Kahaleh MB. Vascular involvement in systemic sclerosis (SSc). Clin Exp Rheumatol. 2004;22(3 Suppl 33):S19-23.

6. Blann AD, Illingworth K, Jayson MI. Mechanisms of endothelial cell damage in systemic sclerosis and Raynaud's phenomenon. J Rheumatol. 1993;20(8):13251330.

7. Mourad JJ, Priollet P, Girerd X, Safar M, Lazareth I, Laurent $\mathrm{S}$. The wall to lumen ratio of the radial artery in patients with Raynaud's phenomenon. J Vasc Res. 1997;34(4):298-305.

8. Hebbar M, Peyrat JP, Hornez L, Hatron PY, Hachulla E, Devulder B. Increased concentrations of the circulating angiogenesis inhibitor endostatin in patients with systemic sclerosis. Arthritis Rheum. 2000;43(4):889-893.

9. Distler O, Del Rosso A, Giacomelli R, Cipriani P, Con- 
forti ML, Guiducci S, Gay RE, et al. Angiogenic and angiostatic factors in systemic sclerosis: increased levels of vascular endothelial growth factor are a feature of the earliest disease stages and are associated with the absence of fingertip ulcers. Arthritis Res. 2002;4(6):R11.

10. van Vugt RM, Kater L, Dijkstra PF, Schardijn GH, Kastelein JJ, Bijlsma JW. The outcome of angiography in patients with Raynaud's phenomenon: an unexpected role for atherosclerosis and hypercholesterolemia. Clin Exp Rheumatol. 2003;21(4):445-450.

11. Henness S, Wigley FM. Current drug therapy for scleroderma and secondary Raynaud's phenomenon: evidencebased review. Curr Opin Rheumatol. 2007;19(6):611618.

12. Bogoch ER, Gross DK. Surgery of the hand in patients with systemic sclerosis: outcomes and considerations. J Rheumatol. 2005;32(4):642-648.

13. Huisstede BM, Hoogvliet P, Paulis WD, van Middelkoop M, Hausman M, Coert JH, Koes BW. Effectiveness of interventions for secondary Raynaud's phenomenon: a systematic review. Arch Phys Med Rehabil. 2011;92(7):1166-1180.
14. Tomaino MM, Goitz RJ, Medsger TA. Surgery for ischemic pain and Raynaud's' phenomenon in scleroderma: a description of treatment protocol and evaluation of results. Microsurgery. 2001;21(3):75-79.

15. Wasserman A, Brahn E. Systemic sclerosis: bilateral improvement of Raynaud's phenomenon with unilateral digital sympathectomy. Semin Arthritis Rheum. 2010;40(2):137-146.

16. Fregene A, Ditmars D, Siddiqui A. Botulinum toxin type A: a treatment option for digital ischemia in patients with Raynaud's phenomenon. J Hand Surg Am. 2009;34(3):446-452.

17. Nevskaya T, Ananieva L, Bykovskaia S, Eremin I, Karandashov E, Khrennikov J, Mach E, et al. Autologous progenitor cell implantation as a novel therapeutic intervention for ischaemic digits in systemic sclerosis. Rheumatology (Oxford). 2009;48(1):61-64.

18. Ishigatsubo $\mathrm{Y}$, Ihata A, Kobayashi H, Hama M, Kirino Y, Ueda A, Takeno M, et al. Therapeutic angiogenesis in patients with systemic sclerosis by autologous transplantation of bone-marrow-derived cells. Mod Rheumatol. 2010;20(3):263-272. 